\title{
State of Matter - Transformationen und Innovationen durch erfahrungsbasierte Werkzeuge gestalten
}

\author{
Jannis Hülsen ${ }^{1}$, Stefan Schwabe ${ }^{2}$, Angelika Trübswetter ${ }^{3}$, Clemens Winkler ${ }^{4}$ \\ 1 j.huelsen@udk-berlin.de \\ ${ }^{2}$ stschwabe@udk-berlin.de \\ ${ }^{3}$ angelika.truebswetter@youse.de \\ ${ }^{4}$ mail@clemenswinkler.com \\ 1,2 Universität der Künste, Berlin, Deutschland \\ ${ }^{3}$ YOUSE GmbH, Berlin/Deutschland \\ ${ }^{4}$ Zürcher Hochschule der Künste, Zürich/Schweiz
}

DOI: 10.14464/awic.v3i0.245

\section{ABSTRACT}

State of Matter (SOM) bietet einen partizipativen und begreifbaren Umgang mit abstrakten und schwer zugänglichen Phänomenen. Zentraler Grundgedanke ist die Möglichkeit der Übersetzung und Verwandlung von aktuell abstrakten und nicht greifbaren Phänomenen, Prozessen und Themen in eine physische Erfahrbarkeit. State of Matter knüpft hier an das Konzept des erfahrungsbasierten Verstehens und Lernens von Lakoff und Johnson an und erzeugt Verständnis für neue Technologien durch Sinneswahrnehmung, Körperbewegung sowie der direkten Interaktion mit der physischen und sozialen Umwelt. Durch physische Interaktionskonzepte und den Umgang mit scheinbar einfachen Materialien wird ein tastender Zugang zu den unterschiedlichen komplexen Feldern und Technologien ermöglicht. Im Dialog von physischer Erfahrung und Geist entsteht so Raum für Kreativität.

In diesem Artikel wird der Fokus eines handlungsbezogenen State of Matter Ansatzes an der Schnittstelle zum Digitalen genauer betrachtet. Durch das Nutzbarmachen geeigneter physischer Materialqualitäten in erfahrungsbasierten Übungen zu digitalen Prozessen, die heute zum einen stark vernetzte Vereinfachungen im Alltag und zum anderen auch starke Abstraktion mit sich bringen, erzeugt State of Matter Kristallisationspunkte in der Erfahrbarkeit individueller Möglichkeitsräume. Hierbei wird darauf geachtet, dass diese Erfahrbarkeit hinzugezogene digitale Methoden und Werkzeuge zur weitreichenden Analyse von gesellschaftlichen und technologischen Entwicklungsprozessen einschließt. Somit wird das 
Workshopformat State of Matter in diesem Artikel als neuartiges Kommunikations- und Gestaltungsformat und als Bindeglied im Digitalen verstanden.

Keywords: State of Matter (SOM), Innovation, Erfahrungsbasierte Werkzeuge, Research through Design

\subsection{EINLEITUNG UND PROBLEMSTELLUNG}

Die fortschreitende Digitalisierung und intelligente Verknüpfung von Systemen (Internet of Things, Blockchains) führen zu einem tiefgreifenden Wandel, der die Art zu leben, zu arbeiten und miteinander zu agieren, bereits grundlegend verändert hat und weiter verändern wird (BMBF, 2015; Schwab, 2016). Die zunehmende Geschwindigkeit und Komplexität technologischer Entwicklungen sowie der oftmals schwer ersichtliche und greifbare Nutzen der Digitalisierung, stellt Unternehmen und Gesellschaft vor die Herausforderung, ihre Innovations- und Adaptionsfähigkeit auch weiter aufrecht zu erhalten (Commerzbank, 2015; Müller-Schöll, 2016). Die heutige umfassende Gestaltbarkeit von Technik schafft einerseits gesellschaftliche Freiheits- und Optionsgewinne, führt aber andererseits sowohl zu neuen Zonen des Nichtwissens als auch zu gesellschaftlichen Orientierungsverlusten, da Sinnzusammenhänge verloren gehen (Blumenberg, 1986). Moderner Technik ist das Merkmal ihrer eigenen Unbestimmtheit auf sehr basaler Ebene eingeschrieben (Gamm \& Hetzel, 2005). Dieses wirft für Gesellschaft, Organisationen und Individuen grundlegende Fragen zu ihrem Welt- und Selbstverständnis auf und trägt Elemente des Unsichtbaren in sich. Denn die Grundlagen digitaler Technologien oder digitaler Informationen selbst - von Flusser (1993) als Undinge bezeichnet - sind für unser menschliches Auge nicht sichtbar; ihre Auswirkungen durchdringen jedoch potentiell jeden Teil unseres Alltags und vermögen unsere physische Umwelt zu verändern. Aus Gestaltungssicht stoßen genau hier digitale Werkzeuge für die Gestaltung digitaler Transformationen an ihre Grenzen:

Flow der Oberflächlichkeit: Durch die Suggestion der permanenten Verfügbarkeit von digitalem Wissen schleicht sich die Gewohnheit ein, sich in einem komfortablen Flow der Oberflächlichkeit aufzuhalten. Die Vielfalt an Informationen und Möglichkeiten verleitet schnell dazu weiter zu scrollen, sobald ein Thema zu kompliziert wird. Diese Gewohnheit wird vor allem dann problematisch, wenn sie sich auch auf Innovationsprozesse überträgt, da hier ein tiefes Eintauchen in die Thematik (Stichwort: Erfahrungsbasiertes Verstehen; Lakoff \& Johnson 2011; Gallese \& Lakoff 2005) erforderlich ist.

Mein Ich im Prozess: Wir befinden uns in einer Rezeption von dem, was Formationen innewohnt, und damit entsteht auch ein neues Aushandeln, was die eigene physische Identität und die eigenen haptischen Erfahrungen angeht. Selbermachen und Erfahren sind ungleich einer Internetrecherche. Es ist wichtig, Teil des Entstehungsprozesses zu werden.

Interaktion mit dem Unsichtbaren: Neue Technologien haben sich weit entfernt von dem, was als Material begreifbar ist, und sind vielmehr in den mathematischen und semantischen Raum 
von Ökonomien übergegangen. Sie sind aus Gestaltungssicht eine große Herausforderung im Sinne von Zugänglichkeit und Sichtbarkeit. Eine besondere Schwierigkeit ist es, die Vieldeutigkeit und Subjektivität einer vermeintlich blinden Wiederholbarkeit (Blumenberg, 1986) zu reflektieren. Aktuell fehlt es an medialen Ausdrucksmitteln und Kommunikationswerkzeugen, die begreifbar sind und schwer greifbare Prozesse physisch zugänglich machen.

State of Matter beschreitet hier einen Weg abseits gewohnter Pfade, um die physische Zugänglichkeit zu schwer greifbaren Prozessen und komplexen neuen Technologien zu ermöglichen. Anknüpfend an Theorien des erfahrungsbasierten Verstehens und Lernens (Lakoff \& Johnson 2011; Gallese \& Lakoff 2005) macht sich State of Matter das metaphorisch angelegte Konzeptsystem unseres Alltagshandelns zunutze und erzeugt Verständnis für neue Technologien durch Sinneswahrnehmung, Körperbewegung sowie der direkten Interaktion mit der physischen und sozialen Umwelt. Denn in diesem Dialog von physischer Erfahrung und unserem Geist entsteht Raum für Kreativität und Innovation.

\subsection{STATE OF MATTER - ZIELE UND GRUNDPRINZIPIEN}

Zentrales Ziel und Untersuchungsgegenstand des explorativen Ansatzes von State of Matter ist es, Möglichkeiten der Übersetzung und Verwandlung von aktuell abstrakten und nicht greifbaren Phänomenen, Prozessen und Themen in eine physische Erfahrbarkeit zu schaffen. Hierbei geht es primär um die Form in den Dingen. Dies schließt sowohl das Dingliche, den elektrisch sensitiven Speicherchip, wie auch das Undingliche, ein darauf aufbauendes Symbol, ein. Gerade hier „besteht die Stärke der Kybernetik darin, dass behauptet wird, dass Eigenschaften und Verhaltensweisen, die wir in der Vergangenheit ausschließlich lebendigem Fleisch und Blut zugeschrieben haben, auch unabhängig von solch spezifischen Materialität realisiert werden können." (Ashby, 1963, S. 117). Die Basis des SOM-Ansatzes ist dabei das Bewusstsein, dass wir Teil einer dynamischen Welt sind, deren Materialien nicht als feste und unverrückbare statische Masse betrachtet werden sollten, sondern als komplexe, sich stetig verändernde Gesamtheit. Damit schließt SOM das oben erwähnte kybernetische Konzept des Dinglichen und der gleichzeitigen Informiertheit mit ein. Die Ausgangshypothese ist, dass durch eine physische Interaktion mit der materiellen Umwelt Komplexität besser vorstellbar gemacht und diskutiert werden kann; hier knüpft SOM an das Design Thinking an.

Als zwei zentrale Grundprinzipien macht sich SOM einerseits die Idee der Prototypisierung und andererseits die Idee von Erklärungsbeispielen, wie wir sie bspw. aus der Physik kennen, zu eigen. Physikalische und chemische Prozesse sind häufig skalierbar und so lassen sich bestimmte Facetten von Prozessen über Materialien mit ähnlichen Prozesseigenschaften darstellen. Das Material für sich und seine (Prozess-) Eigenschaften treten in den Mittelpunkt. Über das Arbeiten in den SOM Workshops mit dem Fokus auf leicht zugängliche, ungefährliche, reaktionsfreudige Substanzen, kommen bspw. Mehl und Wasser zum Einsatz. So kann ein tastender Zugang zu komplexen Themen und Zusammenhängen sowie 
Materialien und Prozessen, die sonst zu groß, zu klein, zu teuer, zu schnell, zu langsam oder zu gefährlich sind, ermöglicht werden. Neben den Materialien selbst, stehen innerhalb des Workshops aber vor allem die Reflexion der Prozesse und Emotionen im Fokus.

\subsection{STATE OF MATTER - VORGEHENSWEISE UND ERKENNTNISSE}

Um die Brücke von der uns meist unbemerkt und unsichtbar umgebenden digitalen medialen Datenwelt um uns herum über technische Infrastrukturen zu gesellschaftlichen Prozessen und Feldern zu schlagen, werden Kriterien des Greifbarmachens skizziert. Diese stammen aus umfangreichen teilnehmenden Beobachtungen (Schöne, 2003; Flick 2007) bei den bisher neun durchgeführten SOM Workshops (Zeitraum von 2014-2017). Diese fanden mit unterschiedlichen Zielgruppen (insgesamt ca. 100 Workshopteilnehmende) in verschiedenen Kontexten statt: vom universitären Umfeld auf Studierendenebene (u.a. UDK Berlin, Kunsthochschule Düsseldorf, Burg Giebichenstein Kunsthochschule Halle) über den Wissenschaftskontext (u.a. MPI Biochemie in Martinsried und Technische Universität Ilmenau), als auch im öffentlichen Umfeld (u.a. Staatliche Museen zu Berlin, Weserburg Museum für moderne Kunst Bremen und im Ausbildungsbereich im Organisationskontext (u.a. Jung von Matt Academy). In einem explorativen Vorgehen adressiert SOM die eingangs skizzierten Problemstellungen von Flow der Oberflächlichkeit, Mein Ich im Prozess und Interaktion mit dem Unsichtbaren und untersucht die Möglichkeiten der Übersetzung und Verwandlung von aktuell abstrakten und nicht greifbaren Phänomenen, Prozessen und Themen in eine physische Erfahrbarkeit zu schaffen. Die Workshop Analyse erfolgte durch teilnehmende Beobachtungen (Grad der Eingebundenheit bspw. bei der Interaktion mit den Materialien; Grad der Irritation etc.) und umfangreiche Dokumentenanalyse (Selbstreflexion der Teilnehmenden über Textproduktionen) in Anlehnung an kontrastierende Methoden der empirischen Sozialforschung (Stamann, Janssen \& Schreier, 2016). Als Synthese der neun Workshops konnten die folgenden SOM Charakteristika extrahiert werden:

1. Rahmenbedingungen für SOM

- Fokus durch Inszenierung von Raum und Reduktion von bereitgestelltem Material

- Geteilte Moderation in „Unterstuit er" und „Beobachter“

2. Interaktion während SOM

- Förderung der Wahrnehmung durch Sinneserfahrungen, die spielerische Interaktion mit Materialien zulassen und Neugierde, Faszination und Flow fördern

- Training von fokussiertem Hinsehen, Fragen stellen und Entscheidungsfähigkeit

- Provokation von Perspektivwechsel (Kontext, Skalierung, ...)

3. Ergebnisse durch SOM

- Identifikation von Grenzthemen und Innovationspotentialen

- Einbezug und Reflexion der eigenen Person, Erfahrungen und Emotionen im Prozess

- Artikulation des Unsichtbaren und Entwickelten/Entdeckten 
Die Analyse der neun Workshops zeigt, dass die Rahmenbedingungen für SOM ein entscheidender Gelingensfaktor sind: Die maximale Beräumung von Mobiliar bzw. vorgegebener Infrastruktur sowie die Reduktion bereitgestellter Materialien und Werkzeuge (bspw. Mehl, Wasser und Pipetten), schaffen Freiraum für Neues, so dass sich die Teilnehmenden dem Komplexen konzentriert aus einer eigens gewählten Perspektive nähern können. Der Moderationskompetenz und Erfahrung der Workshopleitenden kommt ebenfalls eine zentrale Rolle zu, die an vielen Stellen implizit und nur schwer messbar ist. Die Aufgabenteilung in unterstützende und beobachtende Funktion jedoch ist essentiell. Für die Teilnehmenden selbst steht das sich Nähern dynamischer Prozesse mit ihren Sinnen, auch als tastendes Forschen zu bezeichnen (Hill, 2015), wie bspw. einem tanzenden Wassertropfen auf einer Herdplatte seine In-Formiertheit, also eine Erklärbarkeit abzugewinnen, am Anfang des Workshops. Diese Art und Weise der Betrachtung lässt sich mit einer sinnenden Nachdenklichtkeit aus Leonardo Da Vincis Federzeichnungen eines Wasserstrudels als komplexes Phänomen anschaulich beschreiben. Hinzu kommen Spielmethoden und das Nutzen aufkommender Neugierde, wodurch eine intensive Auseinandersetzung mit dem Material erfolgt (siehe Abbildung 1). Bestimmte Materialeigenschaften, wie zum Beispiel Schäumen, Vermischen, Zerstäuben oder Fließen, lassen sich bspw. als Metapher digitaler Prinzipien übertragen, wie Vernetzbarkeit oder Reaktionsgeschwindigkeit von Informationssystemen.

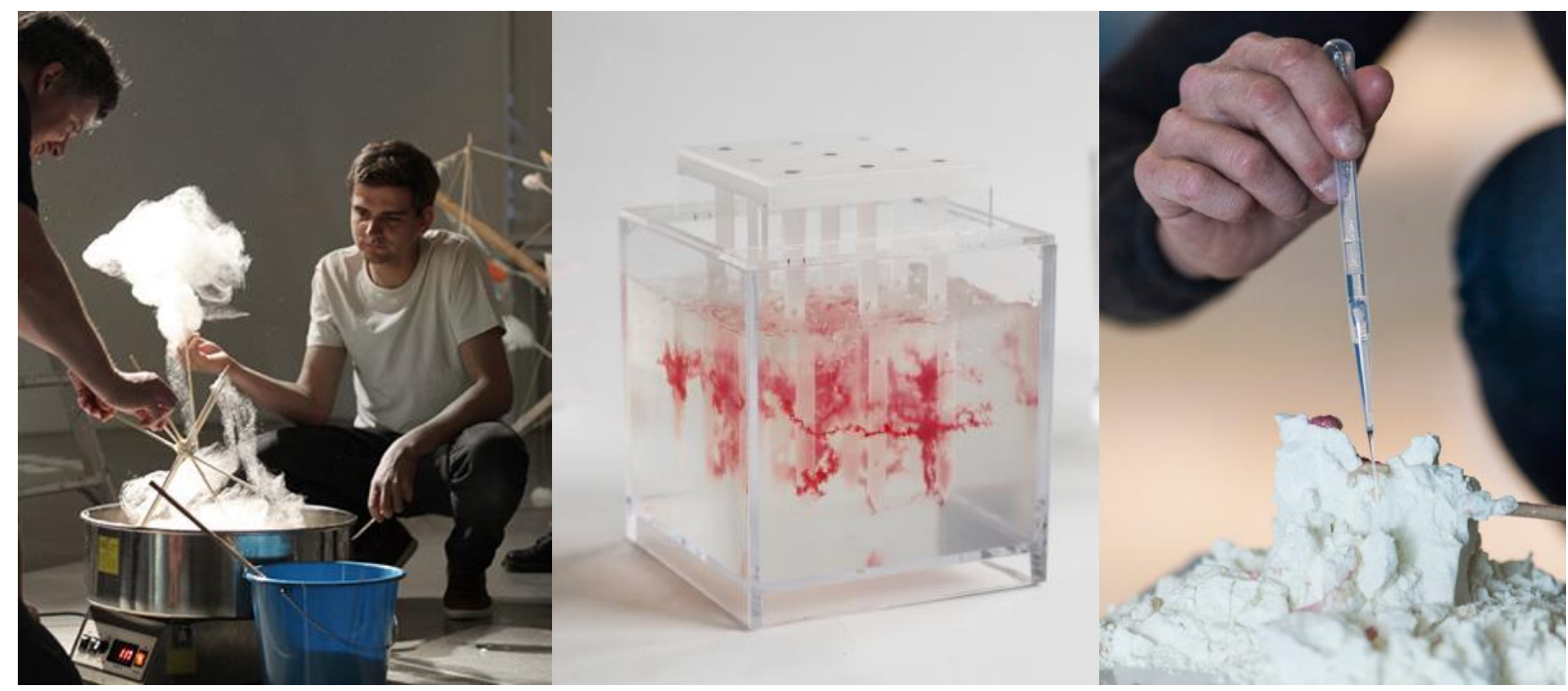

Abbildung 1: $\quad$ State of Matter Anwendungsbeispiele und Workshop Impressionen Quelle: $\quad$ Autor 2, Autor 1, Autor 4 (2017)

Durch den fokussierten Blick bspw. auf den Flug eines Mehlkorns, die Topographien von Wassergräben in Mehllandschaften oder dem Bewegen in Mehlwolken, werden Gefühle des Eintauchens, des Flows (Nakamura \& Csikszentmihalyi, 2014) vermittelt. Wichtig und hilfreich ist in diesem Kontext stets die Frage nach der eigenen Faszination, die gleichzeitig Antrieb für weitere Fragestellungen, sowie sinngebend für das eigene Handeln der Teilnehmenden ist. Intuition, Spielfreude, Neugierde und Faszination ermöglichen Verständnisgewinn und Komplexitätsreduktion und fungieren als zentrale Triebfedern im Innovationsprozess. Dieser 
Aspekt fördert darüber hinaus die Entscheidungskompetenz und liefert durch vertiefende Fragestellungen Grundlagen für ein weiteres Vorgehen. Eine reflektierende Interaktion im Prozess tritt, durch das eigene Aussetzen in diesen räumlich Prozessen, die in der Initiierung meist als Hände schmutzig machen im produktiven Sinne bezeichnet werden können, ein. Vermehrt kommt es zu Fragen: Wie wird kontextabhängig verdinglicht und wie wird informiert? Dies führte auch oft zu Perspektivwechseln. Als Ziel ergeben sich nun Fragen zu Zusammenhängen, Skalierbarkeit und Assoziationsmustern. Ein wichtiger Aspekt ist in dieser Phase die Artikulation zur Übertragung in andere bestehende Disziplinen. Dabei ist einerseits die Frage nach der Reduktion des Inhaltes als Vorstufe der Artikulation ebenso wichtig, wie die Auswahl des Mediums und die Form der Kommunikation. Durch die Notwendigkeit des MitTeilens im SOM Workshop bietet die textuelle, verbalisierte, mediale Ebene stets unerwartete Beobachtungen und Erfahrungen der Teilnehmenden mit dem vermeintlich bereits Vertrauten.

Alles in allem bewegt sich State of Matter bei der Ausbildung und Stärkung jener menschlicher Eigenschaften, die in Unternehmen und Gesellschaft notwendig sind, um die eingangs beschriebene Innovations- und Adaptionsfähigkeit auch im Hinblick auf den digitalen Wandel weiter zu stärken.

\subsection{SYNTHESE UND AUSBLICK}

Seit einigen Jahren zeichnet sich der Trend ab, dass Methoden aus dem Design als Kreativitäts- und Innovationsmethoden auch vermehrt in anderen Disziplinen aufgegriffen werden (Pfeffer, 2014; Fösken, 2015). Kollaborative und interdisziplinäre Ansätze wie Open Innovation oder Design Thinking und Methoden der Zukunftsgestaltung wie Partizipatives Design oder Design Fiction/Speculative Design, gewinnen zunehmend an Bedeutung (Grots \& Creuznacher, 2012). State of Matter adressiert die Herausforderung der innovativen Zukunftsgestaltung, indem es als Workshopformat materialbasierte Methoden und eine damit einhergehende Erfahrbarmachung als Ausgangspunkt für die Entwicklung von neuen Denkräumen, Ideationsstrategien und Produktentwicklung verwendet. Als Designmethode positioniert sich State of Matter im frühen Innovationsprozess und bietet aktuell folgende Einsatzmöglichkeiten:

- als Kommunikationswerkzeug in interdisziplinären Teams

- als Werkzeug für Produktinnovationen

- als Werkzeug für strategische Ideation

- als Werkzeug für das Training von kreativem Denken

- als Kommunikations- und Partizipationswerkzeug bei der Entwicklung disruptiver Technologien (Bsp. Bioökonomie; BMBF-Projekt „Farming the Uncanny Valley“)

Der vorgestellte handlungsbezogene SOM Ansatz wurde in neun Workshops erprobt und analysiert und kann anhand der dargestellten Charakteristika (siehe 1.3) zugänglich gemacht werden. Im Ergebnis zeigt sich, dass SOM Möglichkeiten der Übersetzung und Verwandlung von aktuell abstrakten und nicht greifbaren Phänomenen, Prozessen und Themen in eine 
physische Erfahrbarkeit schafft. Als Forschungsgrenzen sind aktuell zu nennen, dass die Rolle der Moderation noch nicht vollständig expliziert werden kann und keine objektiven Messverfahren zur Untersuchung dieser herangezogen wurden. So ist ein Bias durch unterschiedliche Eigenschaften der Moderation nicht auszuschließen. Ebenfalls noch offen ist die Frage danach, inwiefern Vorwissen, Rückfragen und persönlicher Zugang der Teilnehmenden zu Forschungs- und Innovationsthemen die Qualität der SOM Ergebnisse beeinflusst. Die Rolle von Moderation und Teilnehmenden selbst gilt es daher in einem nächsten Schritt noch stärker in den Untersuchungsfokus zu stellen. Ebenso wenig stand die Vergleichbarkeit der Workshops hinsichtlich Umfang, Teilnehmende und Formate im Zentrum der explorativen Herangehensweise und bietet somit Entwicklungsspielraum. Zudem zeigten sich bei der Untersuchung von Möglichkeiten der Übersetzung und Verwandlung von aktuell abstrakten und nicht greifbaren Phänomenen, Prozessen und Themen in eine physische Erfahrbarkeit erste Grenzen der Skalierbarkeit und Simulierbarkeit bestimmter Phänomene, die es weiter zu untersuchen und zu dokumentieren gilt. Von diesem Forschungsstand ausgehend verspricht SOM jedoch, auch bei der Entwicklung kreativer Digitalisierungsstrategien sowie neuer digitaler Technologien, Produkte und Services eine vielversprechende Methode zu sein. Der Fokus auf erfahrungsbasierte Übungen zu digitalen Prozessen, die ein Spannungsfeld zwischen Vereinfachungen im Alltag und starker Abstraktion im Allgemeinen mit sich bringen, soll SOM Kristallisationspunkte in der Erfahrbarkeit individueller Möglichkeitsräume erzeugen. Durch die Erfahrbarkeit werden auf neue Art und Weise digitale Methoden und Werkzeuge zur weitreichenden Analyse von gesellschaftlichen und technologischen Entwicklungsprozessen eingeschlossen. SOM nähert sich an digitale Prozesse an, baut interdisziplinäre Auseinandersetzungen aus und entwickelt neue Anwendungsfelder. Der Ansatz versteht sich somit als neuartiges Kommunikations- und Gestaltungsformat und als Bindeglied im Digitalen.

\section{LITERATURVERZEICHNIS}

Blumenberg, H. (1986): Wirklichkeiten in denen wir leben, Reclam Verlag.

Bundesministerium für Bildung und Forschung, BMBF (2015): Die fortschreitende Digitalisierung und intelligente Verknüpfung von Systemen, Berlin. URL: https://www.bmbf.de/pub/Industrie_4.0.pdf

Coole, D.; Frost, S. (2010): New Materialisms: Ontology, Agency, and Politics. Duke University Press.

Commerzbank (2015): Management im Wandel: Digitaler, effizienter, flexibler!, Frankfurt am Main. URL: https://blog.commerzbank.de/verantwortung-erleben/2015mai/2015_05_11_fl_up_15_studie_es_neu.pdf

Flick, U. (2007): Einführung in die Qualitative Sozialforschung. Eine Einführung. Rowohlt Taschenbuch Verlag; Auflage: 8, Erweiterte Neuausgabe.

Flusser, V. (1993): Dinge und Undinge: phänomenologische Skizzen 1920-1991. München, Wien: Carl Hanser Verlag. 
Fösken, S. (2015): Innovation braucht systematisch gestaltete Kreativität. URL: http://www.absatzwirtschaft.de/innovation-braucht-systematisch-gestaltetekreativitaet-54771/

Gallese, V.; Lakoff, G. (2005): The Brain's concepts: The Role of The Sensory-Motor System in Conceptual Knowledge. In: COGNITIVE NEUROPSYCHOLOGY, 2005, 21 (0). URL: http://old.unipr.it/arpa/mirror/pubs/pdffiles/Gallese-Lakoff_2005.pdf.

Gamm, G.; Hetzel, A. (2005): Unbestimmtheitssignaturen der Technik. Eine neue Deutung der technisierten Welt. Bielefeld: Transcript Verlag.

Grots, A. \& Creuznacher, I., (2012): Design Thinking — Prozess oder Kultur? Drei Fallbeispiele einer (Veränderungs-)Methode. In: Organisationsentwicklung (2). S.14-21. URL: http://improjects.unikoblenz.de/edschool/downloads/ZOE_Grots_Creuznacher_2_12.pdf

Hill, D. (2014): Dark Matter and Trojan Horses: A Strategic Design Vocabulary", Strelka Press.

Lakoff, G.; Johnson, K. (2011): Leben in Metaphern: Konstruktion und Gebrauch von Sprachbildern, Heidelberg: Carl -Auer Verlag; 7. Auflage.

Nakamura, J; Csikszentmihalyi, M. (2014): The Concept of Flow. In: Csikszentmihalyi, M. (Hrsg.): Flow and the Foundations of Positive Psychology, S. 239-263.

Pfeffer, F. (2014): To Do: Die neue Rolle der Gestaltung in einer veränderten Welt. Strategien | Werkzeuge | Geschäftsmodelle. Ein Kompass zum Erschließen neuen Terrains. Mainz: Verlag Hermann Schmidt.

Ross Ashby (1963): Zitiert nach G. Günther, in: Das Bewusstsein der Maschinen, Günther, G. (Hrsg.), Baden-Baden: Agis Verlag.

Schöne, H. (2003): Die teilnehmende Beobachtung als Datenerhebungsmethode in der Politikwissenschaft. Methodologische Reflexion und Werkstattbericht. Forum Qualitative Sozialforschung, $4 \quad$ (2), URL: http://www.qualitativeresearch.net/index.php/fqs/article/view/720/1559

Stamann, C.; Janssen, M. \& Schreier, M. (2016). Qualitative Inhaltsanalyse - Versuch einer Begriffsbestimmung und Systematisierung. Forum Qualitative Sozialforschung, 17(3), Art. 16, URL: http://nbn-resolving.de/urn:nbn:de:0114-fqs1603166.

Schwab, K. (2016): Die vierte industrielle Revolution. München: Pantheon Verlag. 